Pacific Journal of Mathematics

SOME THEOREMS ON MAPPINGS ONTO 


\title{
SOME THEOREMS ON MAPPINGS ONTO
}

\author{
M. REICHBACH
}

Introduction and summary. Let $F: X \rightarrow Y$ be a continuous mapping of a topological space $X$ into a space $Y$. An important problem is to find conditions ${ }^{1}$ under which this mapping is a mapping onto: $F(X)=Y$. In the present paper, the following consideration is used in proving theorems on mappings onto.

Conditions are given under which the image $F(X)$ is closed and open in $Y$; hence for a connected $Y, F(X)=Y$.

This idea is not new. For instance C. Kuratowski² showed that, i, a subgroup $G$ of a topological additive group $X$ has the Baire propertyf then either $G$ is of the first category in $X$ or $G$ is both open and closed in $X$, so that $G=X$ if $X$ is connected.

It was also used by the author in [10], to obtain some generalizations of the Fundamental Theorem of Algebra.

In this paper the results obtained in [10] are generalized to general topological spaces.

In $\S$ I the notion of a "polynomial mapping" is introduced. Roughly speaking, a mapping $F: X \rightarrow Y$ is called a polynomial mapping if it maps every sequence which does not contain a convergent subsequence onto a sequence which also does not contain a convergent subsequence. It is proved that a polynomial mapping $F: X \rightarrow Y$ of a complete space $X$ into a space $Y$ maps sets closed in $X$ onto sets closed in $Y .^{3}$

The role of the disconnection properties in the proofs of theorems on mappings onto is discussed and a generalization of the Fundamental Theorem of Algebra to $n$-dimensional Euclidean spaces is obtained.

In $\S$ II some theorems on mappings onto are proved for the so-called generalized $F$-spaces and the Fundamental Theorem of Algebra is generalized to such spaces. Finally an application of this generalization to an existence theorem in some class of functional equations is given. For the sake of completeness, many known definitions are recalled.

I, 1. Let $X$ be a space in which convergence satisfying the following two conditions is defined:

$\left(a_{0}\right)$ if $x_{n} \rightarrow x$ and $x_{n} \rightarrow y$ then $x=y$

(a) if $x_{n} \rightarrow x$ and $k_{1}<k_{2}<\cdots$, then $x_{k_{n}} \rightarrow x$

The set of all convergent sequences $\left\{x_{n}\right\} \subset X$ will be denoted by $L$. Note that

Received December 21, 1959.

1 For examples of such conditions see [2], chapter XI.

${ }^{2}$ See [6], p. 38 and [7], p. 81 . Also [4], p. 8.

3 According to the terminology used by Whyburn in $\lceil\mathbf{1 1}]$, we can say that if $F: X \rightarrow Y$ is a polynomial mapping, then it is a strongly closed mapping. 
(b) if $\left\{x_{n}\right\} \in L, k_{1}<k_{2}<\cdots$ and $x_{k_{n}} \rightarrow x$ then $x_{n} \rightarrow x$ Indeed, since $\left\{x_{n}\right\} \in L$, there exists a point $x_{0}$ such that $x_{n} \rightarrow x_{0}$. Hence by (a), $x_{k_{n}} \rightarrow x_{0}$ and since $x_{k_{n}} \rightarrow x$ we obtain by $\left(a_{0}\right), x_{0}=x$. Thus $x_{n} \rightarrow x$.

In the usual way, we can introduce the notions of a subspace $P$ of $X$ and closedness-and-openness in $X$ (or in $P$ ). A set $P \subset X$ is called connected if it is not a union of two non-empty disjoint sets closed in $P$.

Let $C$ be a set of sequences $\left\{x_{n}\right\} \subset X$ such that $L \subset C$. The set $C$ will be called the set of Cauchy (or fundamental) sequences. If $L=C$, the space is called complete. Note that since the definition of $C$ is quite arbitrary (it is only needed that $L \subset C$ ) we can put $C=L$ and the space will be a complete space.

I, 2. A mapping $F: X \rightarrow Y$ of a space $X$ into a space $Y$ is called continuous if for every sequence $\left\{x_{n}\right\}_{n=1,2,}, \ldots \subset$ the condition $x_{n} \rightarrow x$ implies $F\left(x_{n}\right) \rightarrow F(x)^{4}$. By "mappings" we shall in the sequel understand continuous mappings only. We introduce now the following

Definition 1. A sequence $\left\{x_{n}\right\}_{n=1,2, \ldots} ; x_{n} \in X$ is called a non-Cauchy sequence or simply a $N C$ sequence if it does not contain a subsequence belonging to $C$.

If the set of Cauchy sequences is defined as usual, then

(c) in a finite dimensional Banach space the set of $N C$ sequences is identical with the set of sequences $\left\{x_{n}\right\}_{n=1,2, \ldots}$. with $x_{n} \rightarrow \infty$.

Indeed, if $x_{n} \rightarrow \infty$, then by the completeness of the Banach space, $\left\{x_{n}\right\}$ cannot contain a Cauchy sequence $\left\{x_{k_{n}}\right\}$, since otherwise, there would be $x_{k_{n}} \rightarrow x$ for some $x$, which is impossible, by $x_{n} \rightarrow \infty$. On the other hand if $x_{n}$ does not tend to $\infty$, there exists a bounded subsequence $\left\{x_{n}^{\prime}\right\}$ of $\left\{x_{n}\right\}$ and since $X$ is finite dimensional, $\left\{x_{n}^{\prime}\right\}$ contains a convergent subsequence $\left\{x_{k_{n}}^{\prime}\right\}$, which is a Cauchy sequence.

Definition 2. A mapping $F: X \rightarrow Y$ is called a polynomial mapping $^{5}$ if the condition

$$
\left\{x_{n}\right\} \text { is a } N C \text { - sequence, } x_{n} \in X
$$

implies that

$$
\left\{F\left(x_{n}\right)\right\} \text { is a } N C \text { - sequence, } F\left(x_{n}\right) \in Y \text {. }
$$

By (c ) we obtain that

(d) Polynomial mappings $F: X \rightarrow Y$ of a finite dimensional Banach space $X$ into a finite dimensional Banach space $Y$ are identical with

" In fact we should denote the convergence relation in $Y$ by a symbol other than " $\rightarrow$ " used for convergence in $X$, but the meaning of " $\rightarrow$ " will always be clear from the text.

5 The definition of a polynomial mapping was first introduced by the author in [10] for metric spaces. The definition introduced here is a generalization of this definition to general topological spaces. 
those which map sequences $\left\{x_{m}\right\}_{m=1,2}, \ldots$ tending to $\infty$ onto sequences $\left\{F\left(x_{m}\right)\right\}_{m=1,2}, \ldots$ also tending to $\infty$.

In particular, a ( $\neq$ constant) complex polynomial in the complex plane (2-dimensional Banach space) is a polynomial mapping of the plane into itself. This justifies the notion "polynomial mapping".

We prove now the following

Lemma 1. If $F: X \rightarrow Y$ is a polynomial mapping of a complete space $X$ into a space $Y$, then for every set $A$ closed in $X$ the image $F(A)$ is closed in $Y$. (In particular $F(X)$ is closed in $Y$ ).

Proof. Let $y_{n} \in F(A)$ be points belonging to $F(A)$ and let $y_{n} \rightarrow y$. We shall show that $y \in F(A)$. Indeed, there exist points $x_{n} \in A$ such that $y_{n}=F\left(x_{n}\right)$. Now $\left\{x_{n}\right\}$ cannot be a $N C$ - sequence since $\left\{F\left(x_{n}\right)\right\}$ would also be a $N C$ sequence ( $F$ being polynomial mapping) and this is impossible by $L \subset C$ and $y_{n} \rightarrow y$. Therefore, there exists a subsequence $\left\{x_{k_{n}}\right\}$ of $\left\{x_{n}\right\}$ which belongs to $C$. The space $X$ being complete, there is $x_{k_{n}} \rightarrow x$ for some $x$ and $x \in A$ since $A$ is closed. Thus by the continuity of $F, F\left(x_{k_{n}}\right) \rightarrow F(x)$. But $\left\{F\left(x_{k_{n}}\right)\right\}$ is a subsequence of $\left\{y_{n}\right\}$ and therefore by (b) we have $y_{n} \rightarrow F(x)$. Hence by $y_{n} \rightarrow y, F(x)=y$ and by $x \in A$, there is $y \in F(A)$.

Definition 3. A mapping $F: X \rightarrow Y$ is said to be open in the point $y_{0} \in F(X)$ if there exists an open (in $Y$ ) set $U\left(y_{0}\right)$ containing $y_{0}$, such that $U\left(y_{0}\right) \subset F(X)$.

Evidently, $F(X)$ is open in $Y$ if and only if $F: X \rightarrow Y$ is open in every point $y_{0} \in F(X)$.

THeorem 1. If $F: X \rightarrow Y$ is a polynomial mapping of a complete space $X$ into a connected space $Y$, which is open in every point $y \in F(X)$, then $F(X)=Y$. (i.e. $F: X \rightarrow Y$ is a mapping onto).

Proof. By Lemma $1, F(X)$ is closed in $Y$ and by the assumption, $Y-F(X)$ is closed in $Y$. Hence by the connectedeness of $Y$ there is $F(X)=Y$.

I, 3. We shall now investigate the role of the disconnection properties of subsets of $Y$ in the proofs of theorems on mappings onto. Throughout $\S I, 3$ we shall assume that our spaces satisfy the first countability axiom and thus all the topological relations may be expressed in terms of convergent sequences. The following Lemma is evident.

Lemma 2. A mapping $F: X \rightarrow Y$ is not open in the point $y \in F(X)$ if and only if $y \in \operatorname{Fr}(F(X))$, where $\operatorname{Fr}(F(X))$ denotes the boundary of 
$F(X)$ in $Y .^{6}$

We prove now the following

THEOREM 2. If $F: X \rightarrow Y$ is a polynomial mapping of a complete space $X$ into a connected space $Y$ which is open in every point $y \in F(X)-J$, where $J \subset F(X)$ is a set which does not disconnect the space $Y$ and if $F: X \rightarrow Y$ is open in at least one point $y_{0} \in F(X)$, then $F(X)=Y{ }^{7}$

Proof. Since $F: X \rightarrow Y$ is open in the point $y_{0} \in F(X)$ there exists an open (in $Y$ ) set $U\left(y_{0}\right) \subset F(X)$. Denote by $U$ the union of all sets open in $Y$, which are contained in $F(X)$. Evidently $\operatorname{Fr}(U) \subset \operatorname{Fr}(F(X))$. Now suppose, that there would exist a point $x_{0} \in Y-F(X)$ and let $x$ be any point belonging to $U$. Since the set $J$ does not disconnect $Y$ there exists in $Y-J$ a connected set $K$ containing $x_{0}$ and $x$. But the set $\operatorname{Fr}(U)$ disconnect the space $Y$ between $x_{0}$ and $x .^{8}$ Therefore there exists a point $y \in[F r(U)-J] \cap K$ and since $\operatorname{Fr}(U) \subset \operatorname{Fr}(F(X))$ the point $y \in[F r(F X))-J] \cap K \subset F r(F(X))-J$.

By Lemma $1, F(X)$ is closed in $Y$ and therefore $y \in F(X)$. But, by assumption $F: X \rightarrow Y$ is open in every point $y \in F(X)-J$, which by Lemma 2 contradicts the fact that $y \in F r(F(X))-J$. Thus the assumption that there exists a point $x_{0} \in Y-F(X)$ leads to a contradiction.

I, 4. We prove now the

First generalization of the Fundamental Theorem of Algebra. Let $F: X \rightarrow X$ be a mapping of the $n$-dimensional Euclidean space $X$, with $n \geqq 2$ into itself defined by $\eta_{i}=\eta_{i}\left(\xi_{1}, \cdots, \xi_{n}\right) i=1,2, \cdots n$, where the real functions $\eta_{i}$ and their derivatives $\partial \eta_{i} / \partial \xi_{k}$ are continuous in $X$. If then the Jacobian $D=\partial\left(\eta_{1}, \cdots \eta_{n}\right) / \partial\left(\xi_{1}, \cdots, \xi_{n}\right) \neq 0$ in every point $x=$ $x\left(\xi_{1} \cdots \xi_{n}\right) \in X-J_{0}$, where $J_{0}$ is a countable set and if the condition $x_{m}\left(\xi_{1}^{m}, \cdots \xi_{n}^{n}\right) \rightarrow \infty$ implies $F\left(x_{m}\right) \rightarrow \infty$ for every sequence of points $x_{m} \in X$, then $F: X \rightarrow X$ is a mapping onto: $F(X)=X$.

Proof. Since the condition $x_{m} \rightarrow \infty$ implies $F\left(x_{m}\right) \rightarrow \infty$ we obtain by (d) that $F: X \rightarrow X$ is a polynomial mapping. Now by the countability of $J_{0}$ the set $J=F\left(J_{0}\right)$ is also countable and therefore 0 -dimensional. ${ }^{9}$ Hence, since the dimension $n$ of $X$ is $\geqq 2, J$ does not disconnect $X .^{10}$ Further, if $y=F(x)$ is any point of $F(X)-J$, the mapping $F: X \rightarrow X$ is open in $y$, because, by the assumption $D \neq 0$ for points $x \notin J_{0}$, a neighbourhood (in $X$ ) of $y=F(x)$ is covered. Finally, since $J_{0}$ is countable there exists at least one point $y_{0}$ in which $F: X \rightarrow X$ is open. Thus put-

${ }^{6}$ If $X$ is a space and $A$ a subset of $X$, the boundary $\operatorname{Fr}(A)=\bar{A} \cap \overline{X-A}$.

7 This Theorem was suggested to the author by H. Hanani.

8 S. [3], p. 247, also [8], p. 80.

9 In the sense of Menger-Urysohn

10 See [5], p. 48. 
ting $Y=X$ in Theorem 2, we see that all the assumptions of this theorem hold. Therefore $F(X)=X$.

Remark 1. If $F: X \rightarrow X$ is any ( $\neq$ constant) complex polynomial defined on the complex plane $X$, then the mapping $F: X \rightarrow X$ is defined by two real functions $\eta_{1}=\eta_{1}\left(\xi_{1}, \xi_{2}\right), \eta_{2}=\eta_{2}\left(\xi_{1}, \xi_{2}\right)$, where $\eta_{1}$ and $\eta_{2}$ are respectively, the real and imaginary parts of $F(x)=\eta_{1}+i \eta_{2}, x=\xi_{1}+$ $i \xi_{2} \in X, i^{2}=-1$. Now for the Jacobian $D$ there is $D=\left|F^{\prime}(x)\right|^{2} \neq 0$ except for a finite set of points $\left(F^{\prime}(x)\right.$ denotes the derivative of $\left.F(x)\right)$ and therefore, by the above generalization of the Fundamental Theorem of Algebra, $F(X)=X$.

REMARK 2. Our proof of the first generalization of the Fundamental Theorem of Algebra is based on Theorem 2. An essential role in Theorem 2 is played by the assumption that the set $J$ does not disconnect $Y$. This assumption is satisfied because the dimension of the Euclidean space $X$ is assumed to be $\geqq 2$. (A countable set does not disconnect an Euclidean space with dimension $\geqq 2$ ). This explains the role, for the Fundamental Theorem of Algebra, of the fact that the dimension of the Euclidean plane is $\geqq 2$.

II, 1. Let now $X$ be a space (in the sense of I, 1) which is simultaneously a linear space (with multiplication by real or complex numbers). We introduce the following

Definition 4. A mapping $F: X \rightarrow X$ of a linear space into itself is said to have a lower bounded rate of change in the point $y_{0} \in F(X)$ if there exists a point $x_{0} \in F^{-1}\left(y_{0}\right)$, a number (may be complex) $\lambda\left(x_{0}\right) \neq 0$ and an open in $X$ set $U\left(y_{0}\right)$ containing $y_{0}$, such that for every $y^{\prime} \in U\left(y_{0}\right)$ the sequence: $x^{\prime} ; A x^{\prime} ; A A x^{\prime} ; \cdots$ where $A x=x-\lambda\left(x_{0}\right)\left(F(x)-y^{\prime}\right)$ is a Cauchy sequence for some point $x^{\prime} \in X$ (the point $x^{\prime}$ depends on $y^{\prime}$ ).

Lemma 3. If $F: X \rightarrow X$ is a mapping of a complete linear space $X$ into itself, having a lower bounded rate of change in the point $y_{0} \in F(X)$, then $F: X \rightarrow X$ is open in the point $y_{0}$.

Proof. Let $x^{\prime} ; x_{0}, \lambda\left(x_{0}\right) \neq 0$ and $U\left(y_{0}\right)$ be the points, the number and the open (in $X$ ) set defined in the foregoing definition and let $y^{\prime} \in U\left(y_{0}\right)$ be any point of $U\left(y_{0}\right)$. We shall show that $y^{\prime} \in F(X)$. Indeed, since the sequence $x^{-} ; A x^{\prime} ; A A x^{\prime} ; \cdots$ is a Cauchy sequence and $X$ is complete, it has a limit $x_{0}^{\prime}$. Now by the continuity of $A$, we have $A x_{0}^{\prime}=x_{0}^{\prime}$, i.e. $x_{0}^{\prime}-\lambda\left(x_{0}\right)\left(F\left(x_{0}^{\prime}\right)-y^{\prime}\right)=x_{0}^{\prime}$ and hence $F\left(x_{0}^{\prime}\right)=y^{\prime}$.

II, 2. Here we shall introduce the notion of a generalized $F$-space 
and prove some theorems on mappings onto in these spaces. We begin with the definition of a generalized metric space.

Definition 5. A set $X$ of points is called a generalized metric space with metric $\rho$ if on the Cartesian product $X \times X$ a non-negative real function $\rho(x, y)$ is defined: $0 \leqq \rho(x, y) \leqq \infty, x, y \in X$ which satisfies the usual axioms of any metric, i.e. $\rho(x, y)=0$ if and only if $x=y$, $\rho(x, y)=\rho(y, x)$ and $\rho(x, y)+\rho(y, z) \geqq \rho(x, z) ; x, y, z$, belong to $X$.

Thus the difference between the definitions of a metric space and of the generalized metric space consists in the fact that the function $\rho(x, y)$ may assume the value of $\infty$.

ExAmple 1. Take the set $X$ of all real continuous functions $x(t)$, $-\infty<t<\infty$ and define $\rho(x, y)=\sup _{t}|x(t)-y(t)|$. Here, $X$ is a generalized metric space, but not a metric space.

Evidently every metric space is also a generalized metric space. In generalized metric spaces we can define convergent sequences by saying that $x_{n} \rightarrow x$ if $\rho\left(x_{n}, x\right) \rightarrow 0$ and this convergence satisfies $\left(a_{0}\right)$ and (a). Thus every generalized space is a space in the sense of I, 1. If we define, as usual, a Cauchy sequence in $X$ by saying that $\left\{x_{n}\right\}_{n=1,2, \ldots} \in C$ if for every $\varepsilon>0$ there exists a $N(\varepsilon)$ such that $\rho\left(x_{n}, x_{m}\right)<\varepsilon$ for $n, m>N(\varepsilon)$ then we have $L \subset C$. The set $X$ in Example 1 is, as is easy to see, a complete space. Let now $X$ be a generalized metric space which is simultaneously a linear space (with multiplication by real or complex numbers) such that the following two conditions hold

and

( e ) $\rho(x, y)=\rho(x-y, 0)$

(f) $(h x, 0) \leqq|h| . \rho(x, 0)$ for every number $h$ and every point $x \in X$.

In such a case we shall call $X$ a generalized $F$-space. For instance, the space $X$ in Example 1 is a generalized $F$-space. Another example of a generalized $F$ space is the set of all sequences $x=\left(\xi_{1}, \xi_{2}, \cdots\right)$ of real numbers $\xi_{i}, i=1,2, \cdots$ if we define $\rho(x, y)=\sup _{i}\left|\xi_{i}-\eta_{i}\right|$ where $y=\left(\eta_{1}, \eta_{2}, \cdots\right)$. Note that on the set on which $\rho$ is finite, it satisfies the axioms of the so-called $F$-spaces. ${ }^{11}$ This justifies the notion of generalized $F$-space.

Property $T$. Let $F: X \rightarrow X$ be a mapping of a generalized $F$-space into itself such that for the point $y_{0} \in F(X)$ there exists a point $x_{0} \in F^{-1}\left(y_{0}\right)$, a spherical region $S\left(x_{0}, r\right){ }^{12}$ a complex function $\lambda\left(x_{0}\right) \neq 0$ and a real function $\alpha=\alpha\left(x_{0}\right): 0<\alpha<1$ such that for any two points $x$ and $y$

11 See $[\mathbf{1}]$, p. 35 .

12 A spherical region $S\left(x_{0}, r\right)$ with $x_{0}$ as centre and $r$ as radius is defined as the set of all points $x \in X$ satisfying $\rho\left(x_{0}, x\right)<r$. 
belonging to $S\left(x_{0}, r\right)$ there is

(g) $\rho\left[x-y-\lambda\left(x_{0}\right)(F(x)-F(y)), 0\right] \leqq \alpha \rho(x, y)$.

A mapping $F: X \rightarrow X$ for which (g) holds is said to have the property $T$ in the point $y_{0} \in F(X) .^{13}$

We prove now

Lemma 4. If $F: X \rightarrow X$ is a mapping of a generalized $F$-space into itself having the property $T$ in the point $y_{0} \in F(X)$, then $F: X \rightarrow X$ has a lower bounded rate of change in the point $y_{0} \in F(X)$.

Proof. Let $S\left(x_{0}, r\right), \lambda\left(x_{0}\right) \neq 0$ and $\alpha=\alpha\left(x_{0}\right)$ be the spherical region and the functions appearing in the definition of the property $T$ and put $r_{0}=\left[(\Lambda-\alpha) /\left|\lambda\left(x_{0}\right)\right|\right] \cdot r$. It suffices to show that for every $y^{\prime} \in S\left(y_{0}, r_{0}\right)$ the sequence $x_{0}, A x_{0}, A A x_{0}, \cdots$ where $A x=x-\lambda\left(x_{0}\right)\left(F(x)-y^{\prime}\right)$ is a Cauchy sequence: ${ }^{14}$

We have for $x_{1}=A x_{0}$;

$$
\begin{aligned}
& \rho\left(x_{0}, x_{1}\right)=\rho\left(\lambda\left(x_{0}\right)\left(F\left(x_{0}\right)-y^{\prime}\right), 0\right)=\rho\left[\lambda\left(x_{0}\right)\left(y_{0}-y^{\prime}\right), 0\right] \\
& \quad \leqq\left|\lambda\left(x_{0}\right)\right| \rho\left(y_{0}, y^{\prime}\right) \leq\left|\lambda\left(x_{0}\right)\right| \cdot r_{0}=(1-\alpha) r .
\end{aligned}
$$

Thus $\rho\left(x_{0}, x_{1}\right) \leqq(1-\alpha) r$. Now for $x, y \in S\left(x_{0}, r\right)$ we have by $(\mathrm{g})$ :

$$
(\mathrm{g}) ; \rho(A x, A y)=\rho\left(x-y-\lambda\left(x_{0}\right)(F(x)-F(y)), 0\right) \leqq \alpha \rho(x, y)
$$

and therefore $\rho\left(A x_{0}, A x_{1}\right) \leqq \alpha \rho\left(x_{0}, x_{1}\right) \leqq(1-\alpha) \cdot \alpha \cdot r$. Denoting $x_{n}=A x_{n-1}$, $n=1,2, \cdots$ we obtain by induction $\rho\left(x_{n}, x_{n+1}\right) \leqq(1-\alpha) \alpha^{n} r$, hence by $0<\alpha<1$ it is easily seen that $x_{0}, A x_{0}, A A x_{0}, \cdots$ is a Cauchy sequence.

From Lemmas 3 and 4, and from Theorem 2, we obtain the

Second generalization of the Fundamental Theorem of Algebra. If $F: X \rightarrow X$ is a polynomial mapping of a complete generalized $F$-space $X$ into itself, having the property $T$ in every point $y \in F(X)-J$ where $J \subset F(X)$ is a set which does not disconnect the space $X$, and if there exists at least one point $y_{0} \in F(X)$ in which $F: X \rightarrow X$ has the property $T$, then $F(X)=X$.

Proof. By Lemma 4, $F: X \rightarrow X$ has a lower bounded rate of change in every point $y \in F(X)-J$; hence by Lemma $3 F: X \rightarrow X$ is open in every point $y \in F(X)-J$ where by assumption $J$ does not disconnect $X$. Also, by assumption $F: X \rightarrow X$ is open in the point $y_{0} \in F(X)$. Now, since a generalized $F$-space is connected (as a linear space) we see, by putting $Y=X$ in Theorem 2, that the assumptions of this theorem hold. Hence $F(X)=X$.

${ }^{13}$ For some ideas concerning this definition the author is indebted to D. Tamari.

14 The following part of the proof is analogous to the proof of Banach's so-called theorem of contraction mappings, see [9] p. 47 and remark 2 p. 49 . 
REMARK 3. We shall now show that the above theorem is in fact a generalization of the Fundamental Theorem of Algebra. Let $F: X \rightarrow X$ be a ( $\neq$ constant) polynomial, mapping the complex plane $X$ into itself. Since every Banach space is evidently a generalized $F$-space, the complex plane with the usual metric $\rho(x, y)=|x-y|$ is a generalized $F$-space. Now take any point $x_{0} \in X$ such that the derivative $F^{\prime}\left(x_{0}\right) \neq 0$ and let $y_{0}=F\left(x_{0}\right)$. Put $\lambda\left(x_{0}\right)=1 /\left(F^{\prime}\left(x_{0}\right)\right)$ and $\alpha=1 / 2$. Since for $x \rightarrow x_{0}$ we have $\left(F\left(x_{0}\right)-F(x)\right) /\left(x_{0}-x\right) \rightarrow F^{\prime}\left(x_{0}\right)$ there exists a spherical region $S\left(x_{0}, r\right)$ such that for $x, y \simeq S\left(x_{0}, r\right), x \neq y$ there is

$$
\left|\frac{F(x)-F(y)}{x-y} \cdot \frac{1}{\left(F^{\prime}\left(x_{0}\right)\right.}-1\right| \leqq \frac{1}{2}
$$

and therefore $\mid x-y-\left(1 /\left(F^{\prime}\left(x_{0}\right)\right)(F(x)-F(y))|\leqq \alpha| x-y \mid\right.$. But this inequality holds evidently also for $x=y$ and therefore the mapping $F: X \rightarrow X$, defined by the complex polynomial $F(x), x \in X$, has the property $T$ in every point $y_{0}=F\left(x_{0}\right)$ for which $F^{\prime}\left(x_{0}\right) \neq 0$. Now the set $J$ of points $y=F(x)$ for which $F^{\prime}(x)=0$ is finite and thus it does not disconnect the complex plane $X$. Hence by the above second generalization of the Fundamental Theorem of Algebra there is $F(X)=X$, i.e., a complex polynomial maps the complex plane onto itself.

II, 3. It is known that

(h) a $n$-dimensional set does not disconnect the $n+2$-dimensional Euclidean space. ${ }^{15}$

Thus

Lemma 5. A finite dimensional subset $J$ of an infinite dimensional Banach space $X$ does not disconnect $X$.

Proof. Let $x_{0} \in X-J$ be any fixed point and $x$ any point of $X-J$. Suppose that the dimension of $J$ is $n$ and take any $(n+2)$-dimensional plane $E^{n+2}$ (homeomorphic with the Euclidean plane $E^{n+2}$ ) containing the points $x_{0}$ and $x: E^{n+2} \subset X$. Since the set $E^{n+2} \cap J$ is at most $n$-dimensional it does not disconnect $E^{n+2}$ (by (h)) and therefore there exists a connected set $K \subset E^{n+2}-J \subset X-J$ which contains the points $x_{0}$ and $x$. Thus every point $x \in X-J$ may be joined with the point $x_{0} \in X-J$ by a connected set $K \subset X-J$ i.e., the set $X-J$ is connected.

Let now \|\| denote the norm in the Banach space $X$ and define $\rho(x, y)=\|x-y\|$.

We prove the following

THEOREM 3. Let $F: X \rightarrow X$ be a polynomial mapping of an infinite

15 S. [5], p. 48. The term "dimension" is used in the sense of Menger-Urysohn. 
dimensional Banach space $X$ into itself, which maps finite dimensional sets onto finite dimensional sets. If $F: X \rightarrow X$ has the property $T$ in every point $y \in F\left(X-J_{0}\right)$ where $J_{0}$ is a finite dimensional set, then $F(X)=X$.

Proof. Since $J_{0}$ has a finite dimension there exists a point $x_{0} \in X-J_{0}$ and thus the mapping $F: X \rightarrow X$ has the property $T$ in the point $y_{0}=$ $F\left(x_{0}\right)$. Now, the set $J$ of points in which the mapping does not have the property $T$ is contained in $F\left(J_{0}\right)$ and since $F: X \rightarrow X$ maps finite dimensional sets onto finite dimensional sets the set $J$ does not disconnect the space $X$ (by Lemma 5). Hence by the second generalization of the Fundamental Theorem of Algebra there is $F(X)=X$.

Analogously to Lemma 5 it can be proved that

Lemma 6. A 0-dimensional set $J$ does not disconnect a n-dimensional Banach space for $n \geqq 2 .^{16}$

Hence

Theorem 4. Let $F: X \rightarrow X$ be a polynomial mapping of a n-dimensional Banach space $X$ into itself, with $n \geqq 2$. If $F: X \rightarrow X$ has the property $T$ in every point $y \in F\left(X-J_{0}\right)$ where $J_{0}$ is a countable set, then $F(X)=X$.

Proof. For the proof, it suffices to note that the set $F\left(J_{0}\right)$ is countable and thus, by Lemma 6 , does not disconnect the space $X$. The rest of the proof is analogous to that of Theorem 3 and may be left to the reader.

II, 4. An application. Let $X$ be the generalized $F$-space of all real continuous functions $x(t)$ defined on the real line $-\infty<t<\infty$ with metric $\rho(x, y)=\sup _{t}|x(t)-y(t)|$ and let $\phi(t, u)$ be a real continuous function defined for $-\infty<t<\infty,-\infty<u<\infty$ satisfying the conditions:

(i) There exists a real number $m>0$ such that for every pair $u_{1} \geqq u_{2}$ of numbers there is $\phi\left(t, u_{1}\right)-\phi\left(t, u_{2}\right) \geqq m\left(u_{1}-u_{2}\right)$.

(j) For each function $x_{0}(t) \in X$ there exist numbers $r>0$ and $M$ (depending on $x_{0}(t)$ and $r$ ) such that for $x(t)$ and $y(t) \in S\left(x_{0}, r\right)$ there is $\mid \phi(t, x(t))-\phi(t, y(t)|\leqq M| x(t)-y(t) \mid$ for every $t:-\infty<t<\infty$.

Then, the mapping $F(x(t))=\phi(t, x(t))$ maps $X$ onto $X$.

Proof. We shall first show that $F: X \rightarrow X$ is a polynomial mapping. Indeed, we have by (i) $\rho\left(F\left(x_{n}\right), F\left(x_{m}\right)\right) \geqq m \cdot \rho\left(x_{n}, x_{m}\right)$ for every pair $x_{n}(t)$ and $x_{m}(t)$ of functions. Therefore, if the sequence $\left\{F\left(x_{n}\right)\right\}$ would contain

16 This Lemma and Theorem 4 were suggested to the author by $H$. Hanani. 
Cauchy subsequence $\left\{F\left(x_{k_{n}}\right)\right\}$ the sequence $\left\{x_{k_{n}}\right\}$ would be a Cauchy subsequence of the sequence $\left\{x_{n}\right\}$. Thus $F: X \rightarrow X$ maps $N C$ sequences onto $N C$ sequences, i.e., it is a polynomial mapping.

Since our space is complete it suffices, by the second generalization of the Fundamental Theorem of Algebra, to prove that $F: X \rightarrow X$ has the property $T$ in every point $y_{0}=F\left(x_{0}\right) \in F(X)$. Indeed, take any two points $x(t)$ and $y(t)$ belonging to $S\left(x_{0}, r\right)$. Then for $t$ such that $x(t) \geqq y(t)$ we have by (i) and (j) $m(x(t)-y(t)) \leqq F(x(t))-F(y(t)) \leqq M(x(t)-y(t))$, hence $-(m / M)[x(t)-y(t)] \geqq-(1 / M) \cdot[F(x(t))-F(y(t))] \geqq-[x(t)-y(t)]$. Thus $(1-m / M) \cdot[x(t)-y(t)] \geqq x(t)-y(t)-1 / M[F(x(t))-F(y(t))] \geqq 0$. Therefore for any $t$ such that $x(t) \geqq y(t)$ we have

(k) $\quad(1-m / M) \vee|x(t)-y(t)| \geqq|x(t)-y(t)-(1 / M)[F(x(t))-F(y(t))]|$.

Analogously, for any $t$ such that $y(t) \geqq x(t)$, (k) holds and therefore (k) holds for every $t$. Thus assuming that $M>m$ and putting $\lambda=1 / M$ and $\alpha=1-m / M$ we see by (k) that $F: X \rightarrow X$ has the property $T$ in the point $y_{0}=F\left(x_{0}\right)$.

EXAMPLE 2. If $\phi(t, u)$ is a real continuous function defined for $-\infty<t<\infty$ and $-\infty<u<\infty$ having a continuous derivative $\phi_{u}(t, u)$ such that there exist constants $m$ and $M: M>m>0$ for which

$$
m \leqq \phi_{u}(t, u) \leqq M
$$

for every $t$ and $u$, then evidently (i) and (j) hold and hence the function $F(x(t))=\phi(t, x(t))$ maps $X$ onto $X$. Such a function $\phi(t, u)$ is for instance the function $\phi(t, u)=2 u+3 t+\sin (u+t)$.

REMARK 4. An analogous theorem to the above application was proved in [10] for the space $X$ of all real continuous functions $x(t)$ defined in a finite interval $a \leqq t \leqq b^{17}$.

\section{REFERENCES}

1. S. Banach, Theorie des operations linearies, Hafner Publ. Co. (1932), New York.

2. S. Eilenberg, and N. Steenrod, Foundations of algebraic topology, Princeton University Press, (1952), Princeton, New Jersey.

3. F. Hausdorff, Grundzüge der Mengenlehre, (1949), Chelsea Publ. Comp. New York.

4. E. Hille, Functional analysis and semigroups, Amer. Math. Soc., Colloquium Publ. Vol. XXXl, (1948), New York.

5. W. Hurewicz, and H. Wallman, Dimension Theory, Princeton University Press, (1948), Princeton.

6. C. Kuratowski, Sur la propriété de Baire dans les groupes métriques, Studia Math., 4, (1933), 38-40.

7. - Topologie I, Monografie Matematyczne, (1952), Warszawa.

8. —, Topologie II, Monografie Matemtyczne, (1952), Warszawa.

17 See [10], p. 162, Application of Theorem 4. 
9. L. A. Lusternik, and W. J. Sobolew, Elementy funkcjonalnago analiza, (1951), MoskvaLeningrad.

10. M. Reichbach, Generalizations of the fundamental theorem of Algebra, Bulletin of the Research Council of Israel, 7F, (1958), 155-164.

11. G. T. Whyburn, Topological analysis, Princeton University Press, (1958), Princeton, New Jersey.

Department of Mathematics, Technion,

ISRAEL INSTItUTE OF TEChNOLOGY, HaIfA 



\section{PACIFIC JOURNAL OF MATHEMATICS}

\section{EDITORS}

David GILbarg

Stanford University

Stanford, California

\section{F. H. BRowneLL}

University of Washington

Seattle 5, Washington

\section{A. L. Whiteman}

University of Southern California Los Angeles 7, California

\section{J. PAIGe}

University of California

Los Angeles 24, California

\section{ASSOCIATE EDITORS}
E. F. BECKENBACH
T. M. CHERRY
D. DERRY

\author{
E. HEWITT \\ A. HORN \\ L. NACHBIN
}

\author{
M. OHTSUKA \\ H. L. ROYDEN \\ M. M. SCHIFFER
}

E. SPANIER

E. G. STRAUS

F. WOLF

\section{SUPPORTING INSTITUTIONS}

\author{
UNIVERSITY OF BRITISH COLUMBIA \\ CALIFORNIA INSTITUTE OF TECHNOLOGY \\ UNIVERSITY OF CALIFORNIA \\ MONTANA STATE UNIVERSITY \\ UNIVERSITY OF NEVADA \\ NEW MEXICO STATE UNIVERSITY \\ OREGON STATE COLLEGE \\ UNIVERSITY OF OREGON \\ OSAKA UNIVERSITY \\ UNIVERSITY OF SOUTHERN CALIFORNIA
}

\author{
STANFORD UNIVERSITY \\ UNIVERSITY OF TOKYO \\ UNIVERSITY OF UTAH \\ WASHINGTON STATE COLLEGE \\ UNIVERSITY OF WASHINGTON \\ AMERICAN MATHEMATICAL SOCIETY \\ CALIFORNIA RESEARCH CORPORATION \\ HUGHES AIRCRAFT COMPANY \\ SPACE TECHNOLOGY LABORATORIES \\ NAVAL ORDNANCE TEST STATION
}

\footnotetext{
Mathematical papers intended for publication in the Pacific Journal of Mathematics should be typewritten (double spaced), and the author should keep a complete copy. Manuscripts may be sent to any one of the four editors. All other communications to the editors should be addressed to the managing editor, L. J. Paige at the University of California, Los Angeles 24, California.

50 reprints per author of each article are furnished free of charge; additional copies may be obtained at cost in multiples of 50 .
}

The Pacific Journal of Mathematics is published quarterly, in March, June, September, and December. The price per volume (4 numbers) is $\$ 12.00$; single issues, $\$ 3.50$. Back numbers are available. Special price to individual faculty members of supporting institutions and to individual members of the American Mathematical Society: $\$ 4.00$ per volume; single issues, $\$ 1.25$.

Subscriptions, orders for back numbers, and changes of address should be sent to Pacific Journal of Mathematics, 2120 Oxford Street, Berkeley 4, California.

Printed at Kokusai Bunken Insatsusha (International Academic Printing Co., Ltd.), No. 6, 2-chome, Fujimi-cho, Chiyoda-ku, Tokyo, Japan.

PUBLISHED BY PACIFIC JOURNAL OF MATHEMATICS, A NON-PROFIT CORPORATION

The Supporting Institutions listed above contribute to the cost of publication of this Journal, but they are not owners or publishers and have no responsibility for its content or policies. 


\section{Pacific Journal of Mathematics}

\section{Vol. 10, No. $4 \quad$ December, 1960}

M. Altman, An optimum cubically convergent iterative method of inverting a linear bounded operator in Hilbert space . . . . . . . . . . . . . . . . . . . . . . . . . . 1107

Nesmith Cornett Ankeny, Criterion for rth power residuacity ................. 1115

Julius Rubin Blum and David Lee Hanson, On invariant probability measures I . . . . . 1125

Frank Featherstone Bonsall, Positive operators compact in an auxiliary topology ..... 1131

Billy Joe Boyer, Summability of derived conjugate series . . . . . . . . . . . . . . . . 1139

Delmar L. Boyer, A note on a problem of Fuchs . . . . . . . . . . . . . . . . . 1147

Hans-Joachim Bremermann, The envelopes of holomorphy of tube domains in infinite

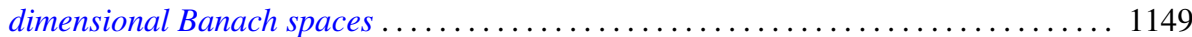

Andrew Michael Bruckner, Minimal superadditive extensions of superadditive

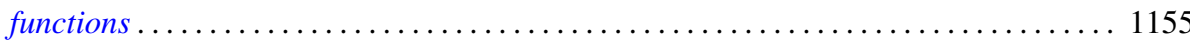

Billy Finney Bryant, On expansive homeomorphisms .................... 1163

Jean W. Butler, On complete and independent sets of operations in finite algebras . . . . . 1169

Lucien Le Cam, An approximation theorem for the Poisson binomial distribution ...... 1181

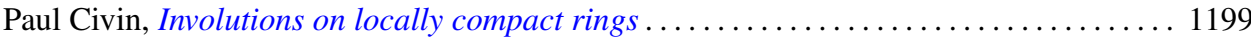

Earl A. Coddington, Normal extensions of formally normal operators . . . . . . . . . 1203

Jacob Feldman, Some classes of equivalent Gaussian processes on an interval ........ 1211

Shaul Foguel, Weak and strong convergence for Markov processes . . . . . . . . . . . 1221

Martin Fox, Some zero sum two-person games with moves in the unit interval ........ 1235

Robert Pertsch Gilbert, Singularities of three-dimensional harmonic functions . . . . . . . 1243

Branko Grünbaum, Partitions of mass-distributions and of convex bodies by

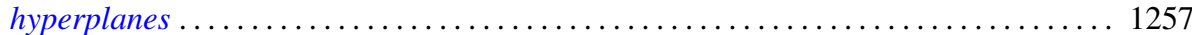

Sidney Morris Harmon, Regular covering surfaces of Riemann surfaces ........... 1263

Edwin Hewitt and Herbert S. Zuckerman, The multiplicative semigroup of integers

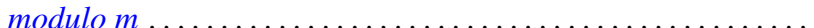

Paul Daniel Hill, Relation of a direct limit group to associated vector groups . ......... 1309

Calvin Virgil Holmes, Commutator groups of monomial groups . .

James Fredrik Jakobsen and W. R. Utz, The non-existence of expansive homeomorphisms

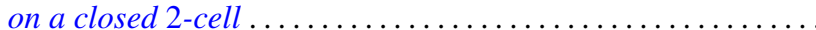

John William Jewett, Multiplication on classes of pseudo-analytic functions . . . . . . . 1323

Helmut Klingen, Analytic automorphisms of bounded symmetric complex domains . . . . 1327

Robert Jacob Koch, Ordered semigroups in partially ordered semigroups . . . . . . . . 1333

Marvin David Marcus and N. A. Khan, On a commutator result of Taussky and

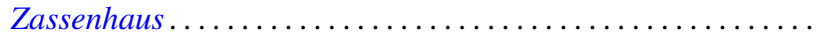

John Glen Marica and Steve Jerome Bryant, Unary algebras......

Edward Peter Merkes and W. T. Scott, On univalence of a continued fraction . . . . . . . 1361

Shu-Teh Chen Moy, Asymptotic properties of derivatives of stationary measures . . . . . 1371

John William Neuberger, Concerning boundary value problems . . . . . . . . . . . 1385

Edward C. Posner, Integral closure of differential rings . . . . . . . . . . . . . . . . . 1393

Marian Reichaw-Reichbach, Some theorems on mappings onto . . . . . . . . . . . . . 1397

Marvin Rosenblum and Harold Widom, Two extremal problems . . . . . . . . . . . . . . . . 1409

Morton Lincoln Slater and Herbert S. Wilf, A class of linear differential-difference

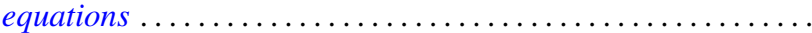

Charles Robson Storey, Jr., The structure of threads . . . . . . . . . . . . . . . . . . 1429

J. François Treves, An estimate for differential polynomials in $\partial / \partial z_{1},, \cdots, \partial / \partial z_{-} n \ldots \ldots 1447$

J. D. Weston, On the representation of operators by convolutions integrals . . . . . . . . 1453

James Victor Whittaker, Normal subgroups of some homeomorphism groups ......... 1469 